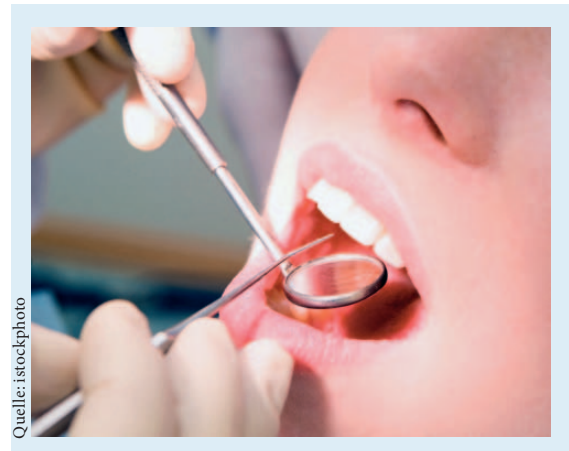

\section{Zahnheilkunde}

Die Zahnmedizin gilt als Domäne des Manuellen. Kein Wunder, denn ein grosser Teil der Tätigkeiten des Zahnarztes ist eher handwerklicher Natur. Dass die Behandlung von Erkrankungen des Gebisses von einem ganzheitlichen Ansatz profitiert, lesen Sie in unserem Expertengespräch auf Seite 193. Und welche Rolle die Akupunktur als wichtiges Instrument in der «Werkzeugkiste» des Zahnarztes spielt, steht direkt nebenan. Einige Zahnärzte haben aus grundsätzlichen Erwägungen eine Abneigung gegen Zahnimplantate; Sie lesen diese auf Seite 197.

\title{
Mit Nadel und Bohrer
}

Zahnärzte können die Akupunktur einsetzen, um ihren Patienten die Angst vor der Behandlung zu nehmen. Sie zeigt auch beim Würgereflex, bei temporomandibularen Funktionsstörungen und bei der Therapie von Gesichtsschmerzen Wirkung. In der Zahnarztpraxis verdient die Akupunktur daher einen festen Platz.

In den letzten 30 Jahren hat sich die Akupunktur zwar nicht als Standardmethode in der Zahnmedizin etablieren können, doch im Hintergrund hat eine ganze Reihe von wissenschaftlichen Untersuchungen ein klares Bild vom Nutzen und von den Grenzen ihres Einsatzes in der Zahnarztpraxis ergeben.

Seit mehr als 3000 Jahren wird sie in China betrieben, und mittlerweile sind viele biochemische Wege aufgeklärt, auf die die Akupunktur wirkt. Längst ist klar, dass sie einen reproduzierbaren Effekt hat, mit dem sich viele postulierte Wirkungen plausibel erklären lassen. Die Akupunktur aktiviert kleine Nervenfasern in den Muskeln, die ein Signal ins Rückenmark senden und dann das Hypophysen-Hypothalamus-System aktivieren. Dort kommt es zur Ausschüttung von z.B. Enkephalinen, beta-Endorphin, Serotonin und Noradrenalin. Indirekt bewirkt die Akupunktur auch die Ausschüttung von Kortisol und trägt somit zur Stärkung des Immunsystems bei. Dort, wo die Nadel gestochen wird, kommt es lokal $\mathrm{zu}$ einer kleinen Entzündungsreaktion, die von der Ausschüttung von Histamin begleitet ist [1].

\section{Einsatz zahlt sich aus}

Aus den zahlreichen Studien lässt sich ableiten, dass die Akupunktur in einigen Bereichen der Zahnmedizin tatsächlich als wertvolle Ergänzung $\mathrm{zu}$ den konventionellen Methoden verwendet werden kann. Sie könnte ihnen teilweise auch überlegen sein. In anderen Bereichen haben sich die Hoffnungen nicht erfüllt, die in die Akupunktur gesetzt wurden. Jetzt, nach drei Jahrzehnten intensiver Forschung vor allem in Grossbritannien und Deutschland, sind die Experten in der Lage, Empfehlungen zur Verwendung der Akupunktur abzugeben. Dabei haben sich vor allem drei Einsatzbereiche herauskristallisiert, in denen der Einsatz der Akupunktur eine sinnvolle Ergänzung zu den konventionellen Methoden ist, was durch Studien auch belegt werden kann: beim Abbau der teilweise starken Angst vor dem Zahnarzt, bei Gesichtsschmerzen, bei der Behandlung von temporomandibularen Funktionsstörungen und des Würgereflexes sowie beim Versuch, nach einer Operation Schmerzen zu lindern.

\section{Die Angst bekämpfen}

Ein Einsatzfeld, bei dem man schon seit langem grosse Hoffnungen in die Akupunktur gesetzt hat, ist die Behandlung von Angstzuständen beim Besuch des Zahnarztes. Zwischen 5-10\% der Bevölkerung haben schwere Angstzustände, wenn sie zum Zahnarzt müssen. Etwa ein Drittel der Menschen geht nicht locker, sondern mit eher leichter Angst und grossem Unbehagen zum Zahnarzt. Auf den ersten Blick scheint die Angst vor dem

\section{KARGER}

Fax +49761 4520714 Information@Karger.d www.karger.com 
Zahnarzt vor allem ein willkommenes Klischee für Witze und Karikaturen zu sein; auf den zweiten Blick hingegen entpuppt sie sich als ein ernst zu nehmendes Problem, das in der Regel gravierende Gesundheitsfolgen bei den Patienten und eine Störung des Praxisablaufs bei den Zahnärzten mit sich bringt. Menschen, die unter Zahnarztangst leiden, verschleppen ihre nötigen Kontrollen, sodass sich ohne die regelmässige zahnärztliche Versorgung die Gesundheitssituation in der Mundhöhle zunehmend verschlechtert. Im schlimmsten Fall müssen sie dann notfallmässig in eine Zahnklinik aufgenommen werden. Dort ist es dann für eine Zahnerhaltung oft zu spät - Zähne müssen gezogen werden, was auf Kosten der Lebensqualität des Patienten geht und oft teuren Zahnersatz notwendig macht. Schlechte Zähne sind auch ein Risikofaktor für Herz-Kreislauf-Erkrankungen. Für den Zahnarzt sind Patienten mit grosser Angst zudem eine Störgrösse im Praxisalltag, die die Organisation beeinträchtigen und auch Kosten verursachen: Aus Angst sagen sie Termine oft im letzten Augenblick $\mathrm{ab}$ und bringen damit Unruhe in die Praxis - von der Frustration, die ausgefallene Behandlung in Rechnung stellen zu müssen und mit den Patienten argumentieren $\mathrm{zu}$ müssen, ganz zu schweigen.

Das Problem der Angst vor dem Zahnarzt ist alt und die Versuche, sie mit verschiedenen Methoden zu bekämpfen, sind vielfältig. Sie reichen von pharmakologischen bis hin $\mathrm{zu}$ ganzheitlichen Ansätzen. Auf der Seite der Pharmakologie wird die Zahnarztangst meistens mit Benzodiazepinen behandelt. Dabei wird Midazolam am häufigsten eingesetzt, da die sedative Wirkung rasch einsetzt und auch schnell wieder nachlässt.

Im Gegensatz zum eher eingeschränkten Angebotsspektrum der Medikamente bietet die Ganzheitsmedizin eine ganz Reihe von Methoden, mit denen Angst gelindert und der Stress vor und beim Zahnarzt-

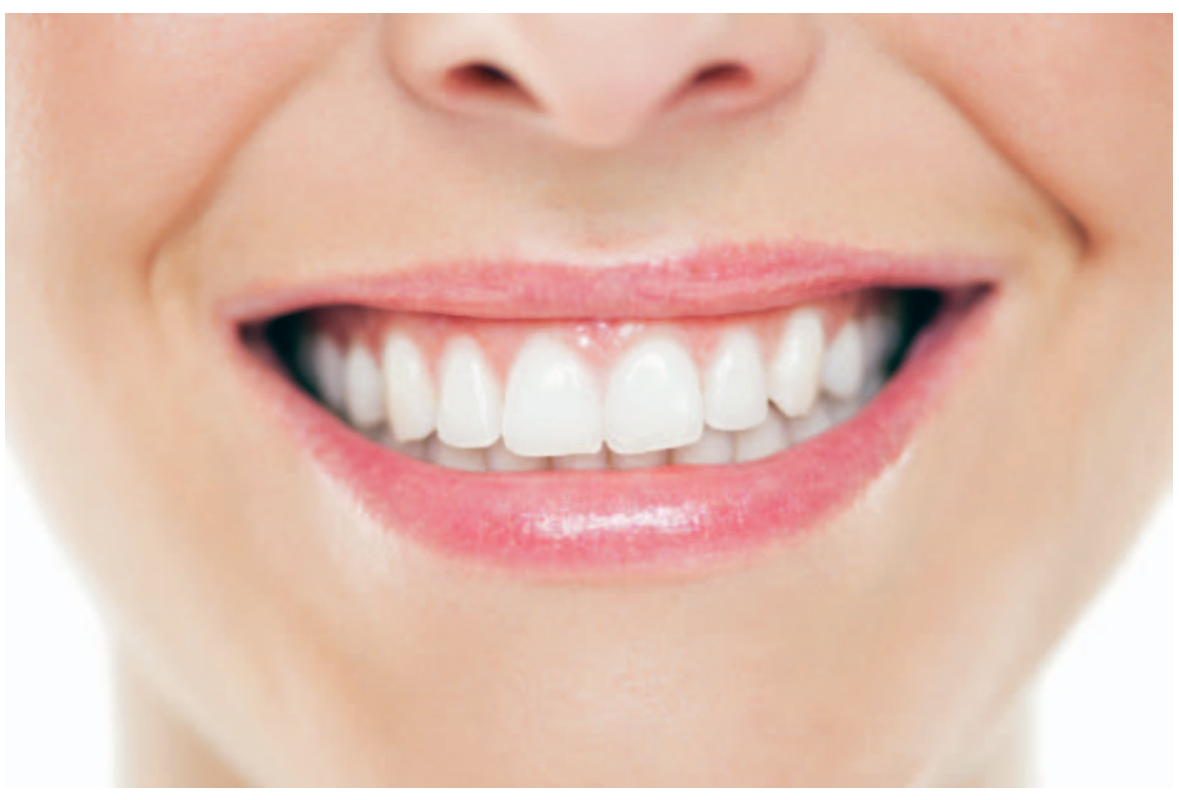

besuch abgebaut werden soll. Dazu gehören z.B. etwa Hypnoseverfahren, Bachblütentherapie oder autogenes Training. Die Entspannungstechniken eignen sich zwar prinzipiell, aber ihr Einsatz in der zahnärztlichen Praxis ist selten realistisch: Dem Zahnarzt und seinem Team fehlen oft die Geduld, sie zu erproben, und schliesslich auch die Zeit, sie einzusetzen. Gesucht ist eine effizientere Lösung, die alltagstauglich ist.

\section{Akupunktur ist einfach zu lernen}

Hier könnte die Akupunktur in $\mathrm{Zu}-$ kunft eine wichtige Rolle in der Zahnmedizin spielen: Mit zwei am Ohr gestochenen Nadeln soll die Angst reduziert werden. Sie eignet sich für die Praxis, denn sie ist einfach anzuwenden und zu lernen. Die «Stechtechnik» und die entsprechenden Einstichstellen für die Angstbehandlung können in einer kurzen Weiterbildung vermittelt werden. In verschiedenen Studien wurde untersucht, ob sich die Methode prinzipiell eignet und ob sie sich hinsichtlich ihrer Wirksamkeit mit den herkömmlichen Behandlungsansätzen vergleichen lässt. Dabei war es vor allem wichtig zu klären, ob sie der Therapie mit Benzodiazepinen ebenbürtig ist. Tatsächlich bietet die Akupunktur ein preiswertes, schnelles und sehr wirksames Mittel, um die Angst vor dem Zahnarzt zu bekämpfen.

Die jüngste Arbeit, die sich mit diesem Thema beschäftigt, stammt von Palle Rosted et al. [2] vom Weston Park Hospital in Sheffield, der sich seit über 20 Jahren mit dem Einsatz der Akupunktur in der Zahnmedizin beschäftigt. Nach seinen Ergebnissen können diese angstgeplagten Patienten aufatmen. In seiner Studie behandelte er 20 Patienten, die zum Teil seit mehr als 30 Jahren unter grosser Angst vor dem nächsten Zahnarztbesuch litten. Die durchschnittlich 40 Jahre alten Patienten zeigten Angstzustände, die mit dem Beck Anxiety Inventory gemessen wurden. Vor der Behandlung lag er im Schnitt bei 26,5 - nach der Akupunktur war er innerhalb kurzer Zeit auf 11,5 gefallen; ein Unterschied, der hochsignifikant war. Bei 70\% der Patienten liess sich durch die Akupunktur eine Verbesserung feststellen.

Der grosse Fortschritt zeigte sich allerdings bei der Betrachtung von Einzelfällen. Von den 20 von Rosted und seinem Team behandelten Patienten waren vor der Akupunktur nur 6 in der Lage, zum Zahnarzt zu gehen. 
Und diese 6 waren so verängstigt, dass es dem Arzt in der Regel nicht möglich war, die Behandlung wie geplant durchzuführen. Für beide, für den Zahnarzt wie auch für den Patienten, war das in der Regel eine sehr unbefriedigende Situation. Nach der Akupunktur hatten alle Patienten ihre Angst im Griff und konnten die Zahnarztbehandlung «in Angriff nehmen». In einer Übersichtsarbeit [1] konnte Rosted zeigen, dass die Wirkung der Akupunktur bei postoperativem Stress in Studien ebenfalls nachgewiesen werden kann.

\section{Besser als Placebo}

Der Einwand, dass die Wirkung der Akupunktur vor allem auf einen Placeboeffekt zurückzuführen ist, ist durch den Aufbau der Studie von Rosted nicht zu entkräften. Es ist seit längerem bekannt, dass bei der Akupunktur ein Teil der Wirkung auf den Placeboeffekt zurückgeführt werden kann. In einer Untersuchung von Karst et al. [3] wurde allerdings für den Placeboeffekt kontrolliert. In ihrer placebokontrollierten Studie, die zum Teil «doppelblind» durchgeführt wurde, beschäftigten sich die Forscher mit der Frage, ob die Wirkung der Akupunktur mit der von Midazolam bei Zahnarztangst vergleichbar ist. Diese Studie ist die erste, die im direkten Vergleich von Akupunktur am Ohr und Midazolam zeigt, dass beide Therapien gleichwertig sind. Karst und sein Team untersuchten knapp 70 Patienten und massen die Angst mit dem Spielberger State-Trait Anxiety Inventory (STAI).

Bei der medikamentösen Behandlung und der Akupunktur setzt die Wirkung gleich schnell nach etwa einer halben Stunde ein; die sedative Wirkung war aber bei der Akupunkturgruppe schneller verflogen. Während bei Midazolam einige Nebenwirkungen beobachtet wurden, zeigten sich solche bei der Akupunkturgruppe nicht; die Akupunktur wurde also deutlich besser vertragen. «Die Ergebnisse dieser Arbeit deuten darauf hin, dass die Akupunktur eine einfache und wirksame Methode ist, um Zahnarztangst abzubauen», meinen Karst und seine Mitarbeiter. Ähnliche Ergebnisse gibt es auch für die Behandlung von Angst mit der Akupunktur vor einer Operation. Dass das «Nadelstechen» beruhigend wirkt, tatsächlich beruhigend wirkt, erstaunt nicht weiter. Alte chinesische Quellen sprechen der Methode eine Stress vermindernde Wirkung zu. Die biochemischen Prozesse, die durch die Akupunkturstiche ausgelöst werden, wirken über die Ausschüttung von Endorphinen direkt auf die Stimmung des Menschen.

\section{Bei Schmerzen wirkungsvoll}

Schon lange wird die Akupunktur bei der Behandlung von Schmerzen eingesetzt, und seitdem die Wirkung auf das Endorphinsystem bekannt ist, ist der Mechanismus nachvollziehbar. Studien über den Einsatz der Akupunktur bei der Behandlung von Schmerzen, die vor allem nach operativen Eingriffen auftreten, gibt es seit mehreren Jahrzehnten in unterschiedlicher Qualität. In einer Übersichtsarbeit versuchte Rosted [4], die aussagekräftigsten $\mathrm{zu}$ analysieren. Er fand, dass bei 11 von 15 Arbeiten, die er genauer betrachtet hatte, die Ergebnisse eindeutig für den Einsatz von Akupunktur sprachen: Dort war die Therapie der Schmerzen effizienter als eine Behandlung mit Placebo oder eine mit konventionellen Mitteln. Allerdings scheint die Wirkung der Akupunktur bei der Anästhesie mit Studien nicht ausreichend belegt zu sein.

\section{Behandlung von Funktionsstörungen}

Bei den temporomandibularen Funktionsstörungen zeigen verschiedene Studien, dass die Akupunktur helfen kann, die Beschwerden in den Griff zu bekommen. Für die Behandlung dieser Funktionsstörung gibt es derzeit keine Standardtherapie, sondern eine Mischung von konventionellen und verschiedenen ganzheitlichen Ansätzen. Die Akupunktur kann hier eine gute Behandlungsmöglichkeit sein. In Literaturübersichten kommen verschiedene Autoren, wie etwa Fink et al. [5], zu dem Schluss, dass in fast $75 \%$ der Arbeiten eine positive Wirkung der Akupunktur festzustellen ist. Auch bei der Behandlung von Gesichtsschmerzen scheint die Akupunktur eine wirksame Methode zu sein.

Bei der Behandlung durch den Zahnarzt ist der Würgereflex des Patienten oft sehr hinderlich. Er macht ein konzentriertes Arbeiten unmöglich, sodass Generationen von Zahnmedizinern nach Methoden gesucht haben, ihn, wenn nicht ganz abzustellen, so doch wenigstens so weit wie möglich zu reduzieren. Wie etwa eine Übersichtsarbeit von Fink et al. [6] zeigt, kann die Akupunktur auch hier sehr hilfreich eingesetzt werden. ( $r f i)$

\section{Literatur}

1 Rosted P: Introduction to acupuncture in dentistry. Br Dent J 2000;189:136-140.

2 Rosted P, Bundgaard M, Gordon S, Pedersen AM: Acupuncture in the management of anxiety related to dental treatment: a case series. Acupunct Med 2010;28:205-206.

3 Karst M, Winterhalter M, Münte S, Francki B, Hondronikos A, Eckardt A, Hoy L, Buhck H, Bernateck M, Fink M: Auricular acupuncture for dental anxiety: a randomized controlled trial. Anesth Analg 2007;104:295-300.

4 Rosted P: The use of acupuncture in dentistry: a systematic review. Acupunct Med 1998;16:4348.

Fink M, Rosted P, Bernateck M, Stiesch-Scholz M, Karst M: Acupuncture in the treatment of painful dysfunction of the temporomandibular joint - a review of the literature. Forsch Komplementmed 2006;13:109-115.

6 Fink M, Stiesch-Scholz M, Karst M: Akupunktur in der Zahnmedizin. Komplementäre Zahnmedizin. ZWR Dtsch Zeitschr Zahnheilkd 2005;114:342-349. 